\title{
SLAP Lesion with Supraglenoid Labral Cyst causing Suprascapular Nerve Compression: A case report
}

\author{
Bryan Y Tan, MBBS (S'pore), Lee KT, FRCS Orth (Ed) \\ Department of Orthopaedic Surgery, Tan Tock Seng Hospital, Singapore
}

\begin{abstract}
We present a case of left suprascapular nerve palsy in a 39year-old female secondary to compression from a large ganglion cyst. She presented with a two month history of left posterior shoulder pain which affected her work and disturbed her sleep. Clinical examination revealed a positive O'Brien's test. Supraspinatus and infraspinatus strength was mildly weakened. A magnetic resonance imaging (MRI) scan of the left shoulder revealed a superior labral anterior to posterior (SLAP) lesion with a large supraglenoid labral cyst extending posteriorly to the spinoglenoid notch, resulting in compression of the suprascapular nerve. Arthroscopy revealed the SLAP lesion with a haemorrhagic labral cyst at the posterosuperior aspect. Arthroscopic decompression of the cyst and SLAP repair with two suture anchors was performed. At 22 months postoperative follow-up, the patient's left shoulder remained asymptomatic with full range of motion and full strength.
\end{abstract}

Key Words:

SLAP lesion; Supraglenoid Labral Cyst; Suprascapular Nerve Compression

\section{INTRODUCTION}

Shoulder pain is among the most common musculoskeletal complaints in the general population. Often, it is due to rotator cuff pathologies such as tendonitis and/or tears, labral pathologies such as superior labral anterior to posterior (SLAP) lesions or instability, capsular pathologies such as adhesive capsulitis, or articular pathologies such as acromioclavicular or glenohumeral arthritis. On rare occasions, suprascapular nerve compression may be the cause of such shoulder pain or weakness. Because of its rarity, this condition is unfortunately often not diagnosed until a magnetic resonance imaging (MRI) scan is performed on the patient who fails to respond to therapy.

\section{CASE REPORT}

We report the case of a 39-year-old female left hand dominant physiotherapist, who presented with a 2-month history of left posterior shoulder pain. The pain was described as dull and aching but severe enough to affect her work and disturb her sleep. She had pain especially during overhead position and internal rotation of the left shoulder. She also complained of mild weakness of her left shoulder. There was no significant injury to her left shoulder but she was active in upper limb sports.

Physical examination revealed a full range of motion of her left shoulder. Pain was elicited during internal rotation to the back and abduction to the overhead position. There was mild weakness of the supraspinatus and infraspinatus muscles as well. In addition, O'Brien's test for SLAP lesion was positive.

An ultrasound of the left shoulder revealed a cyst near the posterior aspect of her left shoulder. An MRI scan of the left shoulder confirmed the presence of a large supraglenoid labral cyst extending posteriorly to the spinoglenoid notch, with a suspected SLAP lesion (Figure 1). This condition most likely resulted in compression of the suprascapular nerve, with resultant early atrophy of the infraspinatus muscle.

Arthroscopy revealed a haemorrhagic labral cyst at the posterosuperior aspect of the labrum (Figure 2), associated with a Type II SLAP lesion (Figure 3). Arthroscopic decompression of the cyst and SLAP repair with 2 suture anchors was performed. The patient's post-operative recovery was uneventful and she was discharged the next morning.

A repeat ultrasound of the left shoulder ten weeks postoperatively confirmed complete resolution of the cyst. At a 22 months follow-up examination, she remained asymptomatic with full range of left shoulder motion and strength. She was able to return to her usual work and sports activities without any restrictions. The Constant Score for her left shoulder was 96 points out of 100 .

\section{DISCUSSION}

The suprascapular nerve may be injured as a result of trauma, repetitive overuse, a space-occupying lesion, or iatrogenic causes. The most common space occupying lesions are

Corresponding Author: Tan Yijia Bryan, Department of Orthopaedic Surgery, Tan Tock Seng Hospital, 11 Jalan Tan Tock Seng, Annex Block Level 4, Singapore 308433

Email: btanyj@hotmail.com 


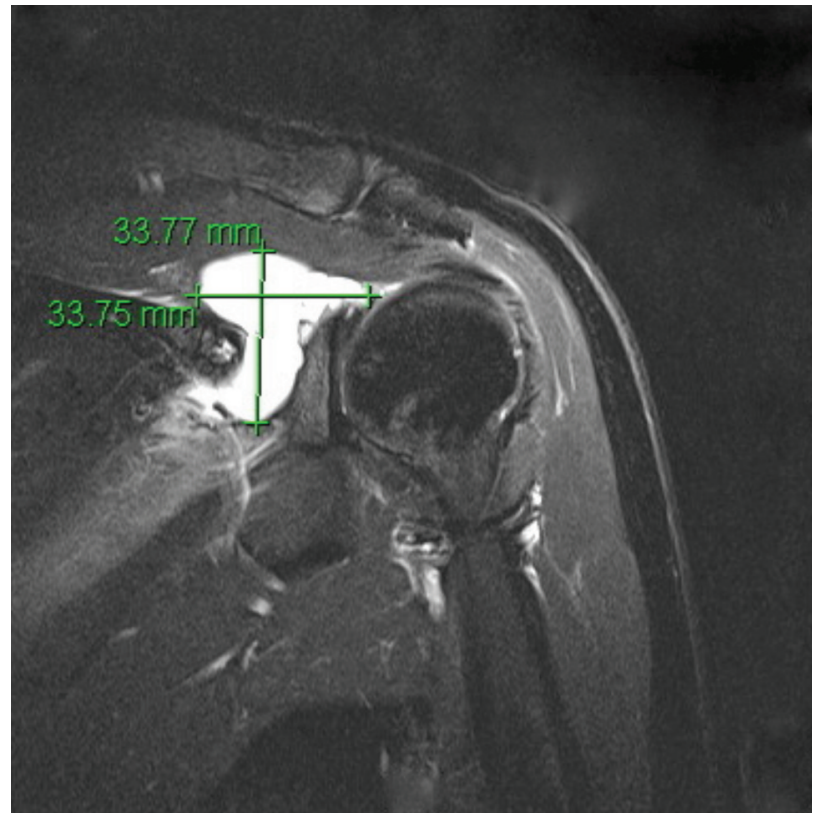

Fig. 1: Magnetic resonance image (MRI) of the left shoulder showing a large supraglenoid labral cyst extending to the spinoglenoid notch, thereby causing suprascapular nerve compression.

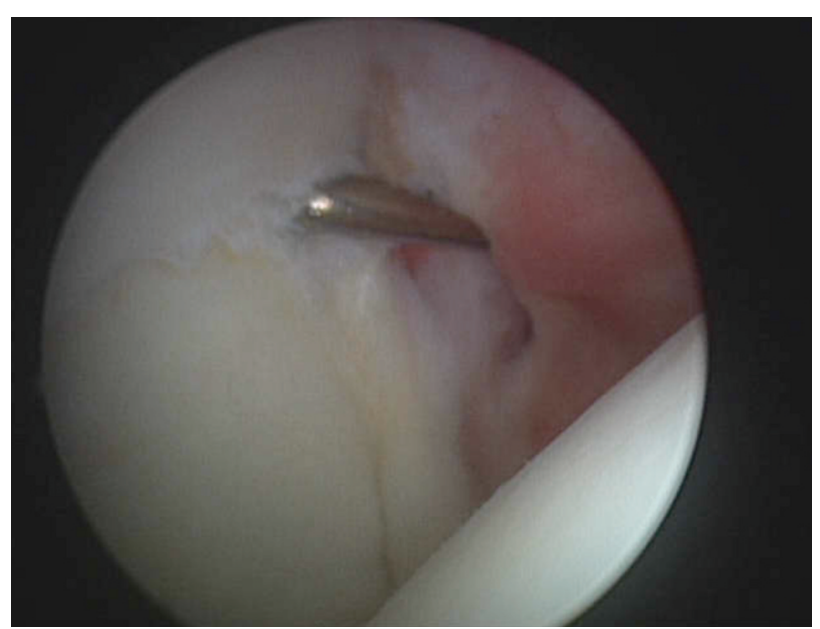

Fig. 3: Arthroscopic image showing a Type II SLAP lesion at the posterosuperior aspect of the labrum.

ganglion cysts arising from the glenohumeral joint. Other masses that have been described include synovial sarcomas, Ewing sarcoma, chondrosarcoma, metastatic renal cell carcinoma, and haematomas associated with an underlying fracture ${ }^{1}$.

The most common presenting symptom is pain, typically located in the posterior aspect of the shoulder, characterised as a dull ache, and exacerbated by overhead activities. A substantial number of patients may present with weakness as the chief symptom, and with little or no pain. Certain patients are completely asymptomatic - in these cases, atrophy may be detected as an incidental finding ${ }^{2}$.

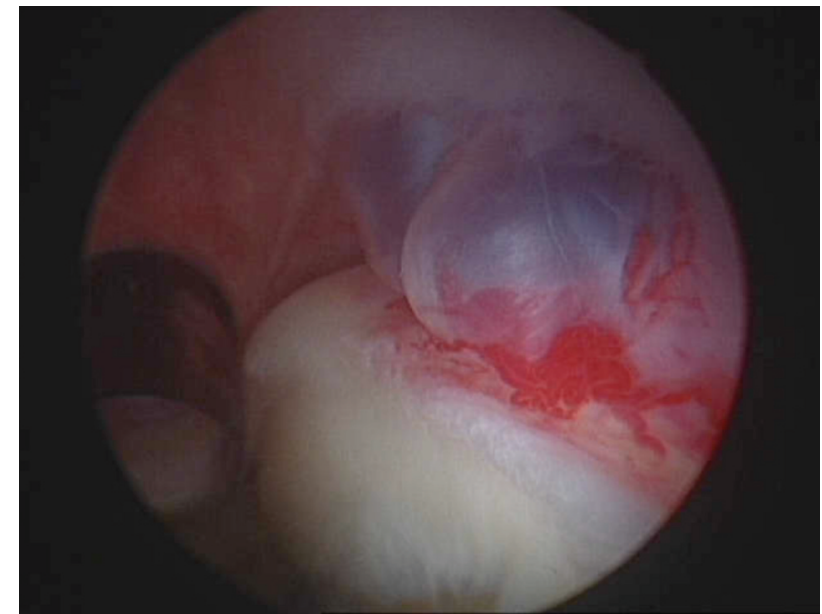

Fig. 2: Arthroscopic image showing a haemorrhagic supraglenoid labral cyst at the posterosuperior aspect of the labrum, with multiple small blood vessels at the posterior rim of the cyst.

There exists a very strong association between ganglion cysts and labral tears. In 1994, Tirman et al. ${ }^{3}$ reported on a study in which all patients with glenoid labral cysts had associated labral tears. This is similar to a 2002 study ${ }^{4}$, which reported that $89 \%$ of MRI-documented ganglion cysts were associated with a labral tear.

What is interesting in our case was the dense haemorrhagic content of the cyst, in contrast to the usual clear synovial fluid. Intraoperatively, the surgeon in fact thought that he might be dealing with an unexpected vascular malformation, and inserted a spinal needle to partially aspirate the cyst first to ensure that it did not refill. The haemorrhage was most likely caused by the rupture of a vessel into the cyst, resulting in rapid expansion in its size and explaining for the huge cyst seen on MRI scan even though the patient only has a short 2-month history of shoulder pain. The presence of multiple small blood vessels at the posterior rim of the cyst (Figure 2) strongly supports this theory.

Treatment of suprascapular nerve entrapment of the shoulder consists of conservative or surgical options. Conservative management usually entails physiotherapy focussed on range of motion and muscle strengthening exercises.

Patients treated by needle aspiration of these cysts have a recurrence rate of up to $48 \%$ in 2 years ${ }^{4}$. In contrast, patients treated with labral defect fixation with or without excision of the cyst report higher satisfaction rates as compared to the non-operative treatment group ${ }^{4,5}$. Patients who underwent surgical excision of the cyst with labral defect fixation report the highest satisfaction of all groups ${ }^{4}$. 
In summary, suprascapular nerve compression secondary to a paralabral ganglion cyst, although uncommon, should form part of the differential diagnosis in a patient who complains of posterior shoulder pain, even in the absence of significant trauma. Because of its frequent association with labral tears, arthroscopic evaluation of the shoulder is important during surgical treatment, and repair of the labral tear is crucial to prevent cyst recurrence and to achieve good long-term outcomes.

\section{REFERENCES}

1. Fritz RC, Helms CA, Steinbach LS, Genant HK. Suprascapular nerve entrapment: evaluation with MR imaging. Radiology. 1992; 182: 437-44.

2. Cummins CA, Messer TM, Nuber GW. Suprascapular nerve entrapment. J Bone Joint Surg Am. 2000; 82: 415-24.

3. Tirman PF, Feller JF, Janzen DL, Peterfy CG, Bergman AG. Association of glenoid labral cysts with labral tears and glenohumeral instability: radiologic findings and clinical significance. Radiology. 1994; 190: 653-8.

4. Piatt BE, Hawkins RJ, Fritz RC, Ho CP, Wolf E, Schickendantz M. Clinical evaluation and treatment of spinoglenoid notch ganglion cysts. J Shoulder Elbow Surg 2002; 11: 600-4

5. Schroder CP, Skare O, Stiris M, Gjengedal E, Uppheim G, Brox JI. Treatment of labral tears with associated spinoglenoid cysts without cyst decompression. J Bone Joint Surg Am. 2008; 90: 523-30. 